the need for further work under standardized experimental conditions.

\author{
J. H. Hendry \\ M. Fox \\ J. M. BoyLE \\ P. T. IYPE
}

\section{New Concepts in Surgical Pathology of} the Skin. R. J. REeD (1976) New York: John Wiley \& Sons. 159 pp. Price $£ 10.90 ; \$ 18.45$.

This rather strange book is the first of a projected series on surgical pathology. The aim of the series is to expound (to quote the series preface) "the why's and how's of specimen handling ", rather than to fortify the diagnostic skills of the hospital pathologist.

Surgical pathology has long been, according to the author, the neglected poor relation of academic pathology in the U.S.A.; worse, " the hospital-based pathologist has favoured the monetarily fruitful specialty of clinical pathology." The state of dermatopathology is even more desperate-as a subspeciality of clinical dermatology, it is seldom accepted as a responsibility of pathologists, nor is it included in pathology training programmes. (This, of course, is all too true of some schools of pathology in Britain, too.)

After drawing such a dark picture in his first chapter, it is not surprising that the author entitles Chapter 2: "Ethics and Deportment ". He describes a battlefield on which clinicians and pathologists struggle for prestige, power, and above all, the patients' dollars. The author gives sound advice on dealing with unscrupulous clinicians, and one might recommend this chapter to British pathologists contemplating flight from the N.H.S.

Later chapters offer sensible, but unecessarily detailed, advice on such elementary topics as the labelling, fixation, and gross description of specimens, the selection of tissue for sectioning and so on.

The last 2 chapters are quite different. One deals with stromal reaction to neoplasia. The other, entitled " Controversies in Dermatopathology" is a rather confused discussion of a variety of topics, including atypical fibroxanthomas, melanomas, and what the author unhappily calls "lymphohistiocytic reticulosis of the skin ". The author suddenly concludes this chapter by telling us that
" clinicians want to be pathologists and pathologists want to be financiers... it appears that the discipline of pathology is being fragmented and dismantled". Can American pathology really be in such a moribund state?

It is hard to know for whom this book has been written. The junior trainees and technicians who might find Chapters 1-4 useful would find Chapters 5-6 very hard going. Practising pathologists would find the earlier chapters banal.

The book is not likely to appeal to pathologists in this country, at least; and at $£ 10.90$ for 159 pages the price is prohibitive. One hopes that later volumes in this series will be more clearly directed towards a specific readership.

\section{O. G. DoDGE}

Cancer, Vol. 3. Biology of Tumours: Cellular Biology and Growth. Ed. F. BECKER (1975) New York and London: Plenum Press. 473 pp. Price $\$ 44.00$

This is the third volume of a series aimed at providing a comprehensive treatise on cancer. The first volume dealt with chemical and physical carcinogenesis and the second with viral carcinogenesis. Volume 3 is concerned with the cell biology of tumours. The underlying theme is to define, and to attempt to explain, the characteristics which make a cell malignant. Topics covered include: potentialities for differentiation in tumour cells; expression of foetal and embryonic genes in tumour cells; the genetics of tumour cells; tumour progression; the physiopathology of solid tumours; energetics; enzyme and metabolic regulation in tumours; control of transcription and translation; protein synthesis in tumour cells; ectopic hormone production; endocrine factors and tumour growth; chalones, and tumour angiogenesis.

Though minor criticisms might be made, on the whole the aim of producing a comprehensive account of tumour biology is successfully achieved. The main themes are treated with clarity and balance, and there are abundant references up to 1975 . This alone would make the book a good investment for research workers in 1976, but in addition to presenting well-ordered and interesting facts, at least some of the contributions give us 
interesting concepts as well. The implications of the views expressed in the chapters on differentiation, embryonic gene expression and angiogenesis have been by no means fully explored, and such ideas may be the basis of a considerable amount of research in future.

The volumes of the series are well produced and indexed, and, considering their size and quality, the price of $\$ 44$ is unusually low: perhaps the publishers are confident of a wide circulation. Their confidence must surely be justified, as Volume 3 can be unreservedly recommended for its wide interest, high quality of writing, up to date factual content, and modest price. It must surely become a standard work in any medical or life science library.

G. W. BAzILL

Advances in Human Tumour Prevention, Detection and Characterization. Vol. 3. Biological Characterization of Human Tumours. Ed. W. DAvis and C. Maltoni (1976) New York: American Elsevier Publishing Co. 418 pp. Price $\$ 55.95$.

This is the sixth of a series of symposia held with the intention of increasing contact between clinicians and laboratory cancer research workers. The papers contributed cover diverse topics and are arranged in sections, which include: "biochemical guide- lines for chemotherapy", " comparison of classical and modern techniques for tumour characterization" and "clinical and experimental studies". None of the papers in the biochemical section contains radically new concepts or information. Discussion of chemotherapy relates principally to established drugs such as methotrexate and alkylating agents and "biochemical guidelines" are not easily discerned. A more systematic approach to tumour biochemistry is described by Weber and colleagues in 2 papers on rat and human hepatomas. Though the qualitative and quantitative changes in enzyme activities described may not be entirely tumour-specific, this approach has the merit of seeking general principles, and it may indicate which enzymes are good targets for attack by chemotherapy.

There are useful reviews of histological and clinical classification of tumours in relation to prognosis and treatment, and 4 papers on the carcinogenicity of vinyl chloride review developments in that area. There is also a fairly extensive discussion of malignant melanoma, in 6 papers which cover epidemiology, characterization and treatment.

This volume contains many contributions of interest, but all in all, it is not an outstanding publication. It is sold in paperback form. 